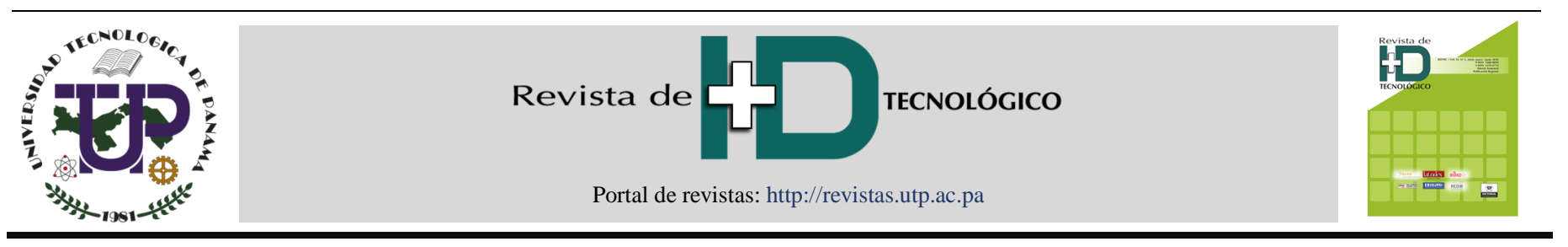

\title{
Respuesta de demanda de energía por introducción de vehículos eléctricos:
}

\section{estado del arte \\ Response of demand for energy by electric vehicles introduction: state of the art}

\author{
Danny Javier Trujillo Sandoval ${ }^{1 *}$, Edwin Marcelo García Torres ${ }^{2}$ \\ ${ }^{1}$ Maestría en Electricidad, Universidad Politécnica Salesiana, Ecuador \\ ${ }^{2}$ Maestría en Electricidad, Universidad Politécnica Salesiana, Ecuador \\ *Autor de correspondencia: dtrujillos2@est.ups.edu.ec
}

RESUMEN-El reemplazo de vehículos basados en combustible fósil por vehículos eléctricos - VE es una necesidad mundial, si bien resulta un gran aporte a la sostenibilidad ambiental y el bienestar de la humanidad lleva consigo grandes retos a todo nivel, entre ellos el estudio del impacto en la demanda de energía eléctrica asociada al consumo de estos vehículos eléctricos. Este documento presenta una revisión bibliográfica asociada con los diferentes temas que aborda esta problemática como son las cifras mundiales de penetración de automóviles eléctricos y el estado de desarrollo actual del elemento clave que son las baterías, sus curvas de carga y descarga. El estudio se realiza a través de una revisión bibliográfica de las bases IEEE Xplore, Science Direct y producción académica relacionada, llegando a identificar varios aspectos claves en la actualidad de los vehículos eléctricos como son las cifras existentes, estimaciones futuras, inversión en infraestructura por países, políticas, entre otros. Se establece también varios criterios asociados al impacto de las características de la batería en el futuro de los VE's a nivel mundial.

Palabras clave- Baterías, estado del arte, energía eléctrica, respuesta a la demanda, vehículo eléctrico.

ABSTRACT - The replacement of vehicles based on fossil fuels with EV - Electric Vehicles is a global necessity, although it is a great contribution to environmental sustainability and the well-being of humanity carries with it great challenges at all levels, such as the study of the impact on the demand of electrical energy associated with the consumption of these electric vehicles. This document presents a bibliographic review associated with the different topics that this problem addresses such as the global data of electric car penetration and the current state of development of its key element: the batteries, along with the loading and unloading curves of the batteries. The study is carried out through a bibliographic review of the IEEE Xplore, Science Direct and related academic production data bases to identify several key aspects of electric vehicles such as existing data, future estimations, percentage of investment in infrastructure by countries and policies, among others. It also establishes several criteria associated with the impact of the characteristics of the battery in the future of EV's worldwide.

Keywords-Batteries, state of the art, electric power, response to the demand, electric vehicle.

\section{Introducción}

La reservas de petróleo a nivel mundial llegarán a agotarse en no más de cinco décadas de acuerdo al ritmo de consumo actual de la población [1], esta situación de disminución de reservas de petróleo y la creciente demanda de países en desarrollo [2] conlleva a la búsqueda de alternativas energéticas [3] que permitan el reemplazo de bienes y servicios asociados con derivados de petróleo entre otras acciones necesarias para afrontar dicha realidad, es así que el sector del transporte al ser uno de los principales motores de desarrollo económico y social [4] ha comenzado hace varios años el reemplazo de vehículos basados en combustible fósil por vehículos eléctricos, siendo esta una de varias estrategias que ya ha sido acogida por la humanidad y se encuentra en ejecución y mejora paulatina de dicha alternativa.

La introducción al mercado de los vehículos eléctricos generará variaciones significativas en la demanda de energía [5] y cambiará las curvas de demanda con las cuales se opera y planifica el sector eléctrico, estos nuevos participantes (carga eléctrica) aumentarán notablemente la elasticidad de la demanda de energía eléctrica residencial [6], por lo tanto, estudios de estimación del cambio en demanda resultan de gran aporte al mercado eléctrico ecuatoriano y a nivel mundial garantizando su fiabilidad [7] y pensando en la flexibilidad del sistema [8].

Con el fin de afrontar el reto, varios estudios ya han comenzado con el análisis de los diferentes elementos que involucran un cambio en la demanda de energía

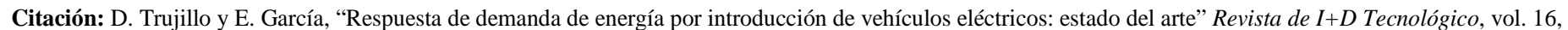
no. 1 , pp. (5-11), 2020.

Tipo de artículo: Original. Recibido: 29 enero de 2019. Recibido con correcciones: 6 abril de 2019. Aceptado: 2 diciembre de 2019.

DOI.

Copyright: 2020 D. Trujillo y E. García. This is an open access article under the CC BY-NC-SA 4.0 license (https://creativecommons.org/licenses/by-nc-sa/4.0/). 
eléctrica, es así que se han aplicado modelos de tipo longitudinal [9], basados en big data [10], patrones de uso aleatorio [6], comportamiento óptimo de estacionamientos [11], juego de gestión de carga [12], modelo Markoviano [13], basado en el modelo de algoritmo Húngaro [14], modelos de predicción estocástica y respuesta a la demanda [15] y modelos propios de los autores en función de las variables de estudio.

En el desarrollo del presente artículo se analizan las cifras del estado actual de penetración de vehículos eléctricos a nivel mundial, las baterías como elemento clave del vehículo eléctrico y como carga a nivel de la demanda energética, de esta forma se brinda una base para estudios de la problemática abordada desde una perspectiva técnica.

\section{Materiales y métodos}

El presente estudio es de tipo descriptivo, mediante una modalidad documental, presenta como problemática principal las bases teóricas que rodean a la penetración de vehículos eléctricos en las sociedades debido a la necesidad de cambio de combustible fósil por energía eléctrica en los sistemas de transporte mundial. Para su desarrollo utiliza revisión bibliográfica de las bases IEEE Xplore, Science Direct, fuentes oficiales y producción académica relacionada a la problemática de penetración de vehículos eléctricos en las sociedades actuales.

Se descompone en dos macro secciones: Escenario actual y prospectivo, y baterías de VE's. En el estudio se presentan las cifras actuales de vehículos eléctricos a nivel mundial y desglosadas por los principales países participantes en el cambio, también se presentan cifras de inversión en infraestructura para cargas eléctricas y finalmente dos escenarios de proyección de cifras al 2030, el primero basado en políticas y el segundo en incorporar las ambiciones prometidas por los países EVI en la Declaración de Campaña EV30 @ 30. Posteriormente se analiza la batería como uno de los elementos claves en el vehículo eléctrico para su futuro en los mercados mundiales, se presentan características importantes de algunas baterías y finalmente curvas de carga y descarga que pueden ser utilizadas para proyectos relacionados.

\section{Resultados
3.1 Penetración de vehículos eléctricos a nivel mundial

La presente sección parte de la base real de vehículos eléctricos en las diferentes sociedades, sus cifras de stock, inversiones y otros aspectos importantes a considerar en la implementación de esta tecnología y su futuro, para esto se desagrega en dos subsecciones: 1 . VE una realidad mundial y 2 . Actualidad y prospectiva del mercado de VE's.

\subsubsection{VE una realidad mundial}

En la actualidad los autos eléctricos son una realidad y cada vez la sociedad va adaptándose a ella, si bien estamos en las fases iniciales de introducción de este tipo de vehículo, en un mediano plazo la introducción de forma masiva de autos eléctricos va a llegar a los diferentes países del mundo e incluirá un aumento significativo de los requisitos de carga de edificaciones y lugares de acceso público solicitando recarga de energía, por lo cual la gestión de respuesta a la demanda es clave [5], [16] y [17] y se debe modelar el VE y estimar dicha sobre demanda [9] con el fin de garantizar el servicio y planificar la expansión del sistema eléctrico de los países [10] [18], además de planificar políticas que permitan optimizar el uso de energía no solo residencial [19], [20] y [21] sino también en los diferentes sectores comerciales e industriales, minimizando los efectos indeseados y cambio de variables como voltajes, frecuencias, etc., en el sistema eléctrico [22] y [23], asociado a esto también se consideran las normas de seguridad que deberán cumplir las estaciones de carga y baterías de VE's [24] entre otros factores.

En el 2017 se pudo observar que a nivel mundial se ha cruzado el umbral de 3 millones de automóviles eléctricos en stock, alrededor de 1.93 millones de BEV's y 1,18 millones de PHVE's [25] y si bien la evolución tiene una curvatura no muy pronunciada, los datos reflejan un crecimiento superior a una característica lineal estimando que en un futuro cercano será de tipo exponencial [25] y [26] debido a la necesidad de cambio mundial del combustible fósil como se puede observar en la figura 1 . 


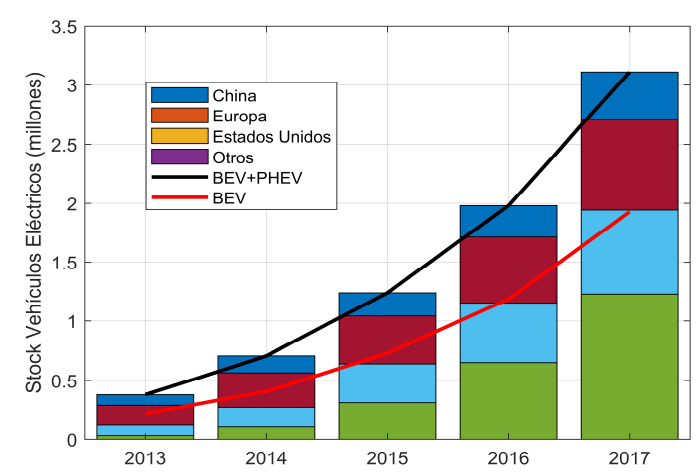

Figura 1. Evolución del stock mundial de vehículos eléctricos, 2013-17 [25].

Estas cifras de cantidad de vehículos eléctricos a nivel mundial no pueden dejar de estar relacionadas con la inversión y planificación de los sistemas eléctricos, políticas gubernamentales, desarrollo tecnológico, entre otros aspectos que sin duda reflejarán cambios sustanciales en las sociedades actuales. Varias iniciativas se han tenido en los últimos años a nivel de los principales países y regiones dando como resultado cifras cada vez mayores de inversión en infraestructura como podemos identificar en la figura 2 .

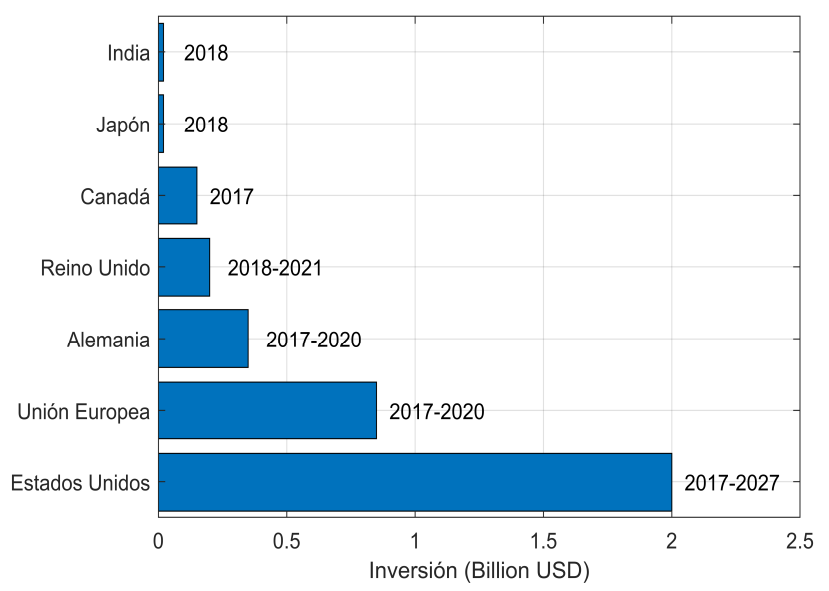

Figura 2. Anuncios de inversión recientes para el desarrollo de infraestructura de vehículos eléctricos en países seleccionados [25].

Uno de los aspectos derivados más importantes de las acciones de inversión se puede observar en la figura 3 , la cual es la infraestructura actual en cifras asociada a puntos de recarga de acceso público clasificados por tipo (cargadores lentos y rápidos) y por país. Se cuenta actualmente con cifras de 318000 estaciones de carga lenta y 112000 estaciones de carga rápida de acceso público a nivel mundial.
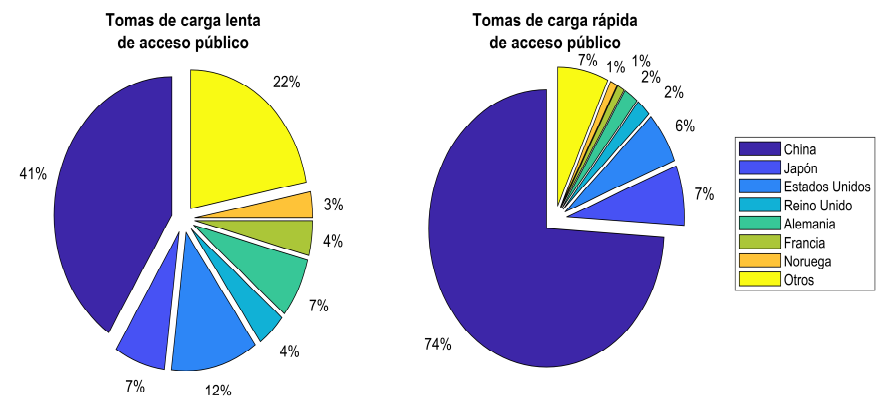

Figura 3. Tomas de carga de acceso público por tipo y país, 2017 [25].

\subsubsection{Actualidad y prospectiva del mercado de VE's}

Como se mencionó anteriormente existen aproximadamente 3,1 millones de VE's de stock a nivel mundial para el año 2017, cifra que podemos observar desglosada en la figura 4 por país y que refleja el esfuerzo y acciones orientadas al cambio de tecnología en automóviles.

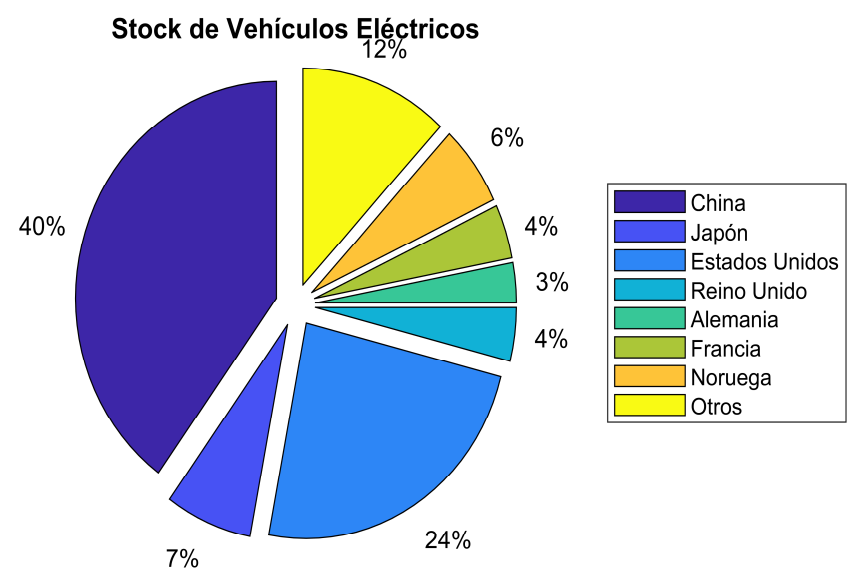

Figura 4. Stock de vehículos eléctricos, 2017 [25].

[25] actualmente ha presentado cifras prospectivas al año 2030 acerca de stock a nivel mundial de VE's, en su estudio analiza dos escenarios utilizando criterios de políticas en el primero y un escenario EV30 @ 30 en el cual incorpora las ambiciones prometidas por los países EVI en la Declaración de Campaña EV30 @ 30 como segundo. En la figura 5 podemos observar una 
adaptación gráfica a los datos proporcionados por [25] con fines ilustrativos para el presente trabajo.

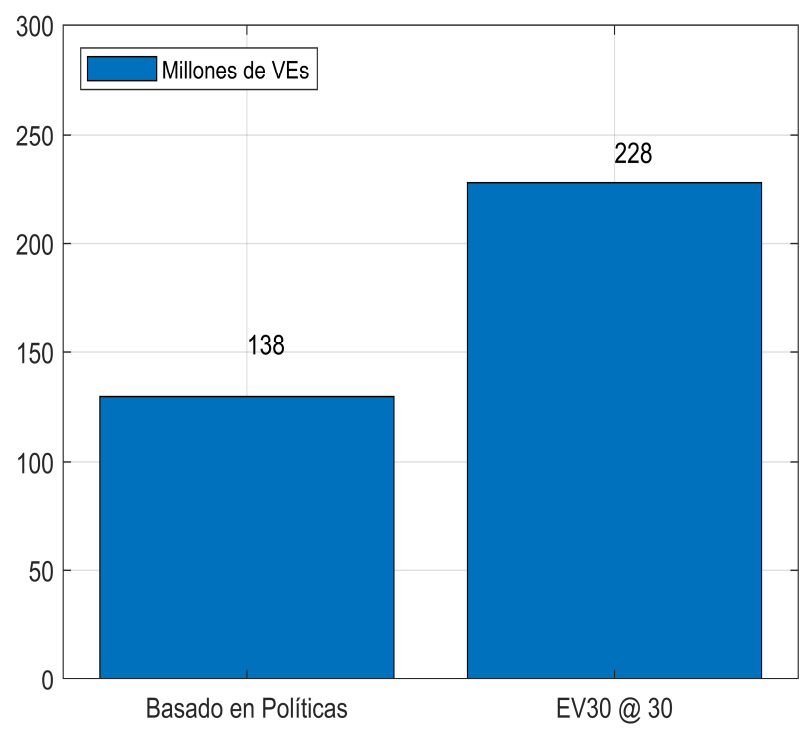

Figura 5. Cifras prospectivas de VE's al 2030.

\subsection{Baterías utilizadas por VE's}

\subsubsection{Baterías - elemento clave de VE's}

Desde el principio de los tiempo la sociedad busca fuentes de energía que permitan mejorar su bienestar [27], en la actualidad cada vez la dependencia de energía eléctrica es mayor en las diferentes actividades de la sociedad [28] y varias de estas dependen directamente de un almacenamiento estacionario como es la batería [29] y [30], por lo cual la gestión de recarga es clave para mitigar los efectos sobre la red eléctrica [31]. Existen varias temáticas asociadas a la problemática de la demanda de energía para vehículos eléctricos, dos de las más importantes es el mercado de reservas [11] y la eficiencia en el uso de los vehículos a través de varios elementos como la capacidad de baterías que si bien ya cuenta con un desarrollo importante llegando a niveles de 60 a $85 \mathrm{KWh}$ todavía está en desarrollo y perspectivas de mejora [32]. Un aspecto importante a considerar en estudios aplicados de demanda energética es el análisis espacial a través de sistemas de información geográfica y métodos estadísticos espaciales [33] pues permitirá una mejor desagregación del problema en diferentes lugares como ciudades, provincias entre otros. También se toma como variable a considerar la carga de baterías de forma estática y dinámica [34] pues resulta de gran aporte tanto en la fase de diseño como en la eficiencia de VE's en campo.

Si bien existen varios métodos ya aplicados como la optimización estocástica [19], [35], [36] y [37] la cual podría ser una importante alternativa que complementado con criterios de precios [37] y [38], análisis de posibles micro redes asociados a la gestión energética [39], características de consumo, tráfico, etc., en la búsqueda de estimación de la demanda, las baterías juegan un papel fundamental y se involucran todos estos métodos, pues son la fuente de energía del auto eléctrico y definen tanto su autonomía como desempeño e implican cambios de consumo energético a nivel del sector eléctrico de cada país, por lo cual es necesario plantear estudios de módulos de recarga, horarios de uso [40] y [41], precio-intervalos de confianza [42], entre otros, para garantizar una optimización en el uso de energía asociado a costos [16] y [43] y una correcta estimación de demanda a futuro. Hay que recordar que la batería es el elemento clave tanto para el vehículo eléctrico como para la red y este elemento es un dispositivo que convierte energía química en energía eléctrica mediante reacción tipo redox y sus principales elementos son: [44]

- Ánodo-electrodo negativo

- Cátodo-electrodo positivo

- Electrolito-conductor iónico.

\subsubsection{Tipos de baterías}

En la actualidad existen varios tipos de baterías utilizadas en automóviles eléctricos y su desarrollo. En cuanto a sus características se ha vuelto una labor constante, pues define en gran parte el servicio que brinda un auto eléctrico en niveles de autonomía, capacidad, seguridad, servicios auxiliares, entre otros [45] y [46]. Los principales tipos de baterías son [47]:

- Baterías de plomo-ácido.

- Baterías de níquel-hierro.

- Baterías alcalinas de magnesio.

- Baterías níquel-cadmio.

- Baterías de níquel-hidruro metálico.

- Baterías de litio.

- Baterías de polímero de litio. 
Existen muchas características que definen una batería y determinan su uso en aplicaciones prácticas [45] algunas esenciales son: su energía específica, su potencia específica y la tensión por celda [48]. En la tabla 1 se puede observar algunas de las principales características de diferentes tipos de baterías que pueden ser utilizados en aplicaciones como el vehículo eléctrico.

Tabla 1. Resumen de las principales características de las baterías más utilizadas en sistemas de tracción eléctrica [47].

\begin{tabular}{|l|l|c|c|c|c|}
\hline Índice & & $\begin{array}{c}\text { Pb- } \\
\text { ácido }\end{array}$ & Ni-Cd & $\begin{array}{c}\text { Ni- } \\
\text { MH }\end{array}$ & $\begin{array}{c}\text { Li- } \\
\text { ion }\end{array}$ \\
\hline $\begin{array}{l}\text { Tensión } \\
\text { nominal }\end{array}$ & $(\mathrm{V} / \mathrm{celda})$ & 2,0 & 1,2 & 1,2 & 4,0 \\
\hline $\begin{array}{l}\text { Energía } \\
\text { específica }\end{array}$ & $(\mathrm{Wh} / \mathrm{kg})$ & 40 & 60 & 90 & 140 \\
\hline $\begin{array}{l}\text { Potencia } \\
\text { específica }\end{array}$ & $(\mathrm{W} / \mathrm{kg})$ & $\begin{array}{c}150- \\
300\end{array}$ & $\begin{array}{c}80- \\
150\end{array}$ & $\begin{array}{c}200- \\
300\end{array}$ & 420 \\
\hline $\begin{array}{l}\text { Densidad de } \\
\text { energía }\end{array}$ & $(\mathrm{Wh} / \mathrm{l})$ & 80 & 95 & 150 & 290 \\
\hline Autodescarga & $(\% / \mathrm{mes})$ & $4-6$ & 10 & $15-25$ & 2 \\
\hline Vida cíclica & $(\mathrm{ciclos})$ & $500-$ & $>1500$ & $>1200$ & - \\
\hline Coste & $(€ / \mathrm{kWh})$ & $120-$ & $250-$ & $300-$ & - \\
& & 150 & 350 & 450 & \\
\hline
\end{tabular}

\subsubsection{Curvas de carga y descarga}

Las curvas características de una batería son uno de los aspectos que definen a la misma, ya que dependiendo de estas se define el tipo de servicio que brindará en sus distintas aplicaciones, en el caso específico de los vehículos eléctricos debido a su funcionalidad se suele clasificar las baterías en función de su tipo de carga considerándolas como tipo de carga: lenta, rápida y ultra rápida, cabe señalar que en cuanto más lenta sea la carga y descarga el rendimiento de la batería será mayor [49], sin embargo el servicio brindado al usuario en cuanto a tiempo podría no ser óptimo. En la figura 6 se puede observar un ejemplo de curvas de carga para baterías de ión-litio de tipo lenta, rápida y ultra rápida.

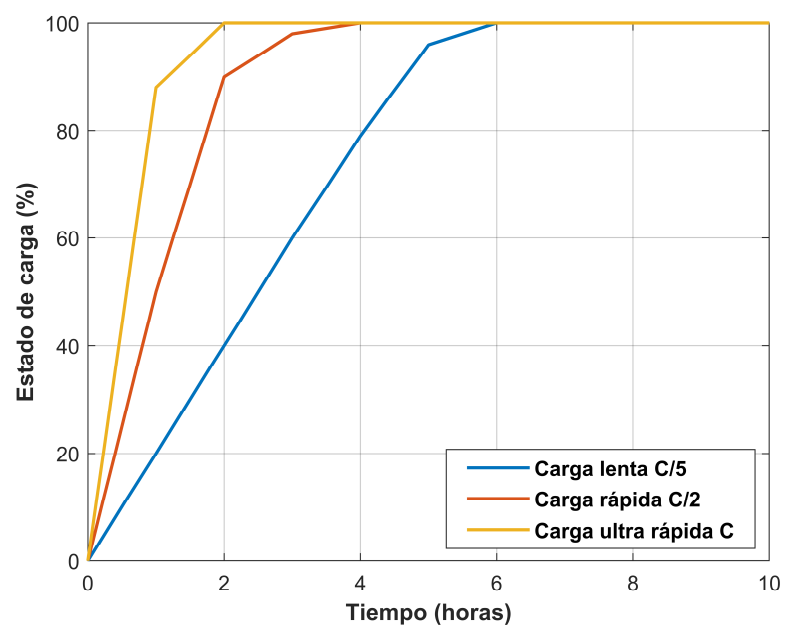

Figura 6. Curvas de carga lenta, rápida y ultra rápida de baterías de ion-Li [50].

De igual forma una curva de descarga para una batería de plomo-ácido se puede identificar en la figura 7 , cabe señalar que las figuras presentadas son casos específicos y deben adaptarse para el tipo de batería a analizar tomando en cuenta sus parámetros propios.

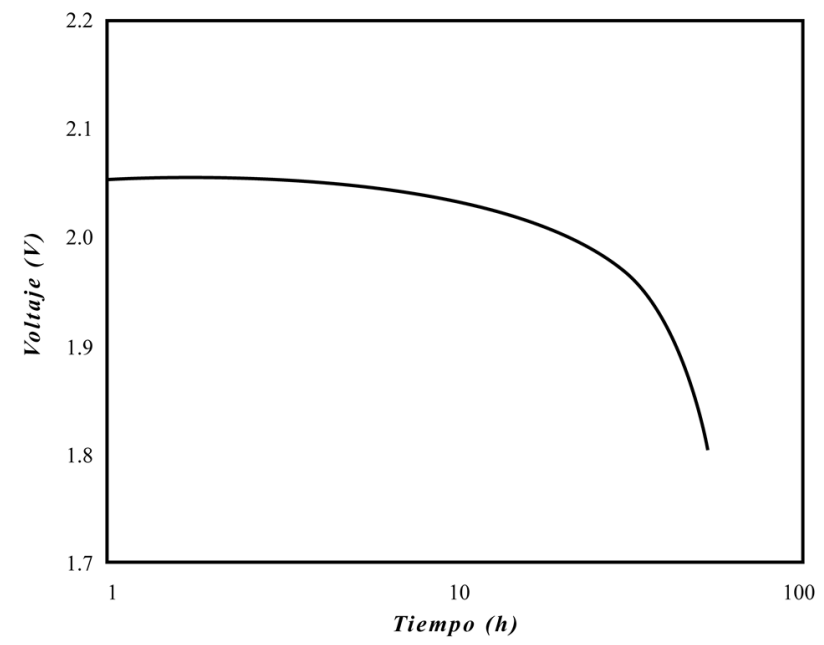

Figura 7. Curva de descarga típica de una batería de plomo ácido [51].

\section{Conclusiones}

La variación de demanda de energía eléctrica a causa de la introducción de vehículos eléctricos es un elemento clave para el desarrollo de esta tecnología, pues si bien ya está desarrollada y operando se encuentra en proceso de mejoramiento y consolidación. El estudio de la demanda de energía eléctrica por tanto 
es vital para la planificación de estaciones de carga, planificación del sistema eléctrico a futuro y de factores asociados a la respuesta de demanda, existen ya diferentes modelos que se han analizado y que presentan una línea base bastante robusta que se deberá adaptar a las realidades de cada región, país o ciudad que requiera solventar dicho escenario.

Dada esta problemática, uno de los elementos claves es la batería, pues sus características y curvas de carga y descarga definen entre varios aspectos la autonomía del vehículo eléctrico definiendo así una de las características más deseables desde la perspectiva del cliente-usuario hacia los VE's. Existe una permanente innovación en baterías lo cual podría resultar beneficioso para futuro de los VE's en los mercados mundiales.

\section{Referencias}

[1] A. Khaligh and Z. Li, "Battery, ultracapacitor, fuel cell, and hybrid energy storage systems for electric, hybrid electric, fuel cell, and plug-in hybrid electric vehicles: State of the art," IEEE Trans. Veh. Technol., vol. 59, no. 6, pp. 28062814, 2010.

[2] E. S. Division, "Assessment of vehicle sizing, energy consumption, and cost through large-scale simulation of advanced vehicle," 2016.

[3] A. Visintin et al., "Presente y futuro de la tecnología de baterías de ion litio : baterías para automóviles eléctricos en la UNLP," 2011.

[4] J. G. Vélez Sánchez, “Análisis y Estimación de la Demanda Eléctrica con la Implementación de Vehículos Eléctricos conectados a una Red de Distribución en Cuenca y El Ecuador," Universidad de Cuenca, 2017.

[5] K. N. Kumar and K. J. Tseng, "Impact of demand response management on chargeability of electric vehicles," Energy, vol. 111, pp. 190-196, 2016.

[6] F. Rassaei, W. S. Soh, and K. C. Chua, "Demand Response for Residential Electric Vehicles with Random Usage Patterns in Smart Grids," IEEE Trans. Sustain. Energy, vol. 6, no. 4, pp. 1367-1376, 2015.

[7] W. Hoiles and V. Krishnamurthy, "Nonparametric demand forecasting and detection of energy aware consumers," IEEE Trans. Smart Grid, vol. 6, no. 2, pp. 695-704, 2015.

[8] D. Papadaskalopoulos and G. Strbac, "Decentralized Participation of Flexible Demand in Electricity Markets Part II: Application With Electric Vehicles and Heat Pump Systems," IEEE Trans. Power Syst., vol. 28, no. 4, pp. 3658-3666, 2013.

[9] J. Asamer, A. Graser, B. Heilmann, and M. Ruthmair, "Sensitivity analysis for energy demand estimation of electric vehicles," Transp. Res. Part D Transp. Environ., vol. 46, pp. 182-199, 2016.
[10] M. B. Arias and S. Bae, "Electric vehicle charging demand forecasting model based on big data technologies," Appl. Energy, vol. 183, pp. 327-339, 2016.

[11] M. Shafie-Khah et al., "Optimal Behavior of Electric Vehicle Parking Lots as Demand Response Aggregation Agents," IEEE Trans. Smart Grid, vol. 7, no. 6, pp. 26542665, 2016

[12] N. Z. Xu and C. Y. Chung, "Challenges in Future Competition of Electric Vehicle Charging Management and Solutions," IEEE Trans. Smart Grid, vol. 6, no. 3, pp. 1323-1331, 2015.

[13] G. Ayala and M. Garcia, "Respuesta De La Demanda Eléctrica Basado En El Modelo Markoviano," Tesis, p. 27, 2016.

[14] R. Baxter, N. Hastings, A. Law, and E. J. . Glass, "Óptima asignación de recursos energéticos para respuesta de la demanda por vehículos eléctricos usando algoritmo húngaro," Anim. Genet., vol. 39, no. 5, pp. 561-563, 2008.

[15] E. Marcelo and G. Torres, "Optimal demand response for the recharging of electric vehicles in micro-networks type of service station by allocation of energy resources," 2018 Int. Conf. Inf. Syst. Comput. Sci., pp. 150-157, 2018.

[16] Y. Xiong, B. Wang, C. cheng Chu, and R. Gadh, "Vehicle grid integration for demand response with mixture user model and decentralized optimization," Appl. Energy, vol. 231, no. September, pp. 481-493, 2018.

[17] S. S. Reka and V. Ramesh, "Demand response scheme with electricity market prices for residential sector using stochastic dynamic optimization," 2016 - Bienn. Int. Conf. Power Energy Syst. Towar. Sustain. Energy, PESTSE 2016, 2016.

[18] A. Morales-Acevedo, "Forecasting future energy demand: Electrical energy in Mexico as an example case," Energy Procedia, vol. 57, pp. 782-790, 2014.

[19] D. Huo, C. Gu, G. Yang, and S. Le Blond, "Combined domestic demand response and energy hub optimisation with renewable generation uncertainty," Energy Procedia, vol. 142, pp. 1985-1990, 2017.

[20] F. McLoughlin, A. Duffy, and M. Conlon, "Characterising domestic electricity consumption patterns by dwelling and occupant socio-economic variables: An Irish case study," Energy Build., vol. 48, pp. 240-248, 2012.

[21] E. Garcia and I. Isaac, "Demand response systems for integrating energy storage batteries for residential users," 2016 IEEE Ecuador Tech. Chapters Meet. ETCM 2016, 2016.

[22] P. Moreno and E. M. Garcia Torres, "Gestión energética mediante procesos estoscásticos para la respuesta a la demanda," p. 26, 2017.

[23] M. J. Vahid-Pakdel, S. Nojavan, B. Mohammadi-ivatloo, and K. Zare, "Stochastic optimization of energy hub operation with consideration of thermal energy market and demand response," Energy Convers. Manag., vol. 145, pp. 117-128, 2017.

[24] B. Wang, P. Dehghanian, S. Wang, and M. Mitolo, 
"Electrical Safety Considerations in Large-Scale Electric Vehicle Charging Stations," IEEE Trans. Ind. Appl., vol. PP, no. c, p. 1, 2019.

[25] I. E. A. International and E. Agency, "Global EV Outlook 2018," 2018.

[26] R. S. Rabié Durán, "Datos para pruebas de carga y descarga de baterías de ion-litio," 2013.

[27] S. Bijani Chiquero, "Electrodeposición y Caracterización de Láminas de $\mathrm{Cu} 2 \mathrm{O}$. Aplicación como Electrodos de Baterías de Ión-Litio," 2007.

[28] J. Martínez Bertran, "Métodos de estimación del estado de carga de baterías electroquímicas," 2017.

[29] J. Guembe Zabaleta, "Cálculo del estado de carga en baterías de plomo-ácido: diseño y validación experimental," 2016.

[30] J. I. Pérez Valderrama and Y. M. Walteros Parra, "Sistema de gestión de carga para baterías de ion-litio," Pontificia Universidad Javeriana-Colombia, 2016.

[31] C. C. Mendoza, "Algoritmo de gestión para la recarga de vehículos eléctricos,” pp. 90-98, 2015.

[32] F. Rassaei, W. S. Soh, and K. C. Chua, "Distributed Scalable Autonomous Market-Based Demand Response via Residential Plug-In Electric Vehicles in Smart Grids," IEEE Trans. Smart Grid, vol. 9, no. 4, pp. 3281-3290, 2018.

[33] H. Tyralisa, N. Mamassis, and Y. Photis, "Spatial analysis of the electrical energy demand in Greece," Energy Policy, pp. 340-352, 2017.

[34] D. Niculae, M. Iordache, M. Ieee, M. Stanculescu, M. Ieee, and M. L. Bobaru, "A Review of Electric Vehicles Charging Technologies Stationary and Dynamic," pp. 9-12, 2019.

[35] A. Z. Moghadam, J. Saebi, and H. J. Dasht Bayaz, "Stochastic optimization of demand response aggregators in wholesale electricity markets," 30th Power Syst. Conf. PSC 2015, no. November, pp. 234-240, 2017.

[36] Y. Iino, T. Hatanaka, and M. Fujita, "Model predictive control for demand response aggregation management system with response delay time and uncertainty model," Proc. SICE Annu. Conf., pp. 1478-1483, 2014.

[37] Z. Chen, S. Member, L. Wu, and Y. Fu, "Real-Time PriceBased Demand Response Management for Residential Appliances via Stochastic Optimization and Robust Optimization," vol. 3, no. 4, pp. 1822-1831, 2012.

[38] X. Yan, Y. Ozturk, Z. Hu, and Y. Song, "A review on price-driven residential demand response," Renew. Sustain. Energy Rev., vol. 96, no. June, pp. 411-419, 2018.

[39] Y. Wang et al., "Energy management of smart micro-grid with response loads and distributed generation considering demand response," J. Clean. Prod., vol. 197, pp. 10691083, 2018.

[40] T. Müller and D. Möst, "Demand Response Potential: Available when Needed?," Energy Policy, vol. 115, no. February 2017, pp. 181-198, 2018.

[41] N. Forouzandehmehr, M. Esmalifalak, H. Mohsenian-Rad, and Z. Han, "Autonomous demand response using stochastic differential games," IEEE Trans. Smart Grid, vol. 6, no. 1, pp. 291-300, 2015.

[42] P. B. Luh, L. D. Michel, P. Friedland, C. Guan, and Y. Wang, "Load forecasting and demand response," IEEE PES Gen. Meet. PES 2010, pp. 1-3, 2010.

[43] L. Jia and L. Tong, "Optimal pricing for residential demand response: A stochastic optimization approach," 2012 50th Annu. Allert. Conf. Commun. Control. Comput. Allert. 2012, pp. 1879-1884, 2012.

[44] R. Iglesias et al., "Modelado y Simulación de una Batería de Ion-Litio Comercial Multicelda," no. May 2014, 2012.

[45] C. Peña Ordoñez, "Estudio de baterías para vehículos eléctricos.," 2011.

[46] A. Torres López, "Sistema de carga con paneles solares para baterías de autos eléctricos," Instituto Politécnico Nacional, 2013.

[47] J. C. Viera Pérez, Carga rápida de baterías de ni-cd y nimh de media y gran capacidad. análisis, síntesis y comparación de nuevos métodos. 2003.

[48] Á. I. Rodríguez Cea, "Modelo para la batería de ion de litio de un vehículo eléctrico," 2017.

[49] C. Morente Fernández, "Análisis y modelado del comportamiento de baterías para vehículos eléctricos," Universidad Carlos III de Madrid, 2015.

[50] B. D. Benalcázar López, "Óptima respuesta a la demanda para estaciones de carga de vehículos eléctricos con alta incertidumbre considerando el perfil de voltaje en la red de distribución," 2017.

[51] J. Balenzategui, "Aproximación al Modelado de los Procesos de Carga y Descarga en Baterías Electroquímicas mediante Ecuaciones Integrales,” 1999. 